\title{
Dissociative Sticking Probability of Methane on $\mathrm{Pt}(110)-(2 \times 1)$
}

\author{
I. F. Peludhero, A. Gutiérrez-González, W. Dong, R. D. Beck,* and H. F. Busnengo*
}

Cite This: https://doi.org/10.1021/acs.jpcc.1c00807

Read Online

ACCESS

Llll Metrics \& More

Article Recommendations

Supporting Information

ABSTRACT: In this work, we revisit the dissociative sticking of methane on $\mathrm{Pt}(110)-(2 \times 1)$ using quasi-classical trajectory (QCT) calculations and supersonic molecular beam (SMB) experiments. Experimentally, we apply the King and Wells method and the reflection absorption infrared spectroscopy (RAIRS) technique to measure the initial dissociative sticking probability, $S_{0}$. Our QCT calculations make use of a reactive force field (RFF) based on density functional theory (DFT) total energy results. We compare our QCT results for $S_{0}$ with experiments and with density functional molecular dynamics (DFMD) data available for $\mathrm{CHD}_{3}(v=0)$. The fact that our QCT RFF-based approach is computationally much cheaper than DFMD allows us to integrate a much larger number of trajectories for longer interaction times. Thus, we can significantly extend the previously reported comparison of QCT-DFMD and experimental results, for $\mathrm{CHD}_{3}(v=0), \mathrm{CH}_{4}(v=0)$, and $\mathrm{CH}_{4}\left(\nu_{3}=1\right)$ to lower incident energies, $E_{\mathrm{i}}(\geq 0.2 \mathrm{eV})$, and surface temperatures, $T_{\mathrm{s}}$ (down to $120 \mathrm{~K}$ ). Our QCT results and the SMB experimental data agree qualitatively with theory, underestimating the experimental

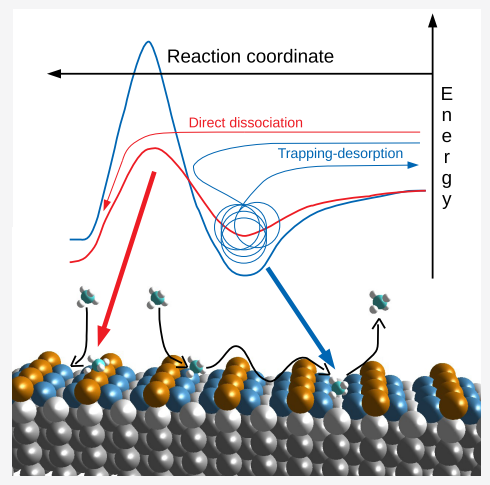
results by a factor of $\sim 2-3$. Our calculations shed light on the fate of the surprisingly large fraction of methane molecules, which remain trapped on the surface for much more than 1 ps (and therefore can hardly be studied using DFMD) for $E_{\mathrm{i}}$ values as large as $\sim 1 \mathrm{eV}$. We show that the contribution of trapped molecules to $S_{0}$ is negligible over a wide range of initial conditions, due to two reasons: (i) the barrier for dissociation is larger than that for desorption on all surface sites and (ii) trapped molecules spend most of the time on top of the valley $\mathrm{Pt}$ atoms, where the physisorption well is the deepest but the energy barrier for dissociation is the highest.

\section{INTRODUCTION}

Dissociative chemisorption of methane is known to be the ratelimiting step of the heterogeneous catalytic steam reforming process used in industry to produce molecular hydrogen. ${ }^{1}$ Due to its importance, the study of this reaction has attracted a great deal of attention from both the experimental and the theoretical sides. $^{2-7}$ Whereas initially most studies were focused on flat low-Miller-index surfaces, more recent work also investigated surfaces that include low-coordinated metal atoms, which resemble the most active sites of real catalysts used in industry. ${ }^{8-16}$ One example is $\operatorname{Pt}(110)-(2 \times 1)$ which, with its missing-row reconstruction, exposes three kinds of surface atoms with coordination numbers 7,9 , and 11 , hereafter referred to as the ridge, facet and valley, respectively (see Figure 1a).

Supersonic molecular beam (SMB) experiments have shown that for impact energies, $E_{\mathrm{i}} \gtrsim 0.1 \mathrm{eV}$, the initial reactive sticking probability, $S_{0}$, of methane on $\mathrm{Pt}(110)-(2 \times 1)$ sharply increases with increasing $E_{\mathrm{i}}{ }^{9}$ Such strong activation by initial translational energy is evidence for a direct reaction mechanism characterized by the bond breaking occurring promptly near the impact site. In addition, state-resolved SMB experiments ${ }^{9}$ showed that methane dissociation on $\mathrm{Pt}(110)$ $(2 \times 1)$ is also activated by vibrational excitation of the incident molecule as well as by surface temperature, $T_{s}$, ${ }^{8}$ similar to what has been found on other metal surfaces for which it is well established that the first $\mathrm{C}-\mathrm{H}$ bond cleavage process proceeds through a fast direct mechanism: e.g., $\mathrm{Ni}(111)^{17-20}$ and $\operatorname{Pt}(111) .^{21,22}$

From the theoretical side, the first calculations of $S_{0}$ for $\mathrm{CH}_{4} / \mathrm{Pt}(110)-(2 \times 1)$ were made by Jackson and co-workers using their reaction path Hamiltonian ( $\mathrm{RPH}$ ) method in combination with quantum dynamics (wavepacket) simulations. ${ }^{23}$ The $\mathrm{RPH}$ method describes the approach of a molecule on a fixed surface site (well justified for a direct lateral-steering-free mechanism) and for a fixed lattice distortion. For comparison with experiments, the $S_{0}$ values obtained for different surface sites and lattice distortions are averaged according to the surface geometry and surface temperature. $^{23}$ Using the $\mathrm{RPH}$ approach for $E_{\mathrm{i}}>0.1 \mathrm{eV}$, Jackson and co-workers obtained results in qualitative agreement with experiments ${ }^{8}$ for translational and vibrational activation as well as for changes in $T_{\mathrm{s}}$ from 400 to $600 \mathrm{~K}$.

More recently, the groups of Beck and Kroes revisited the methane/Pt(110)-(2×1) system $^{24}$ and compared the new

Received: January 28, 2021

Revised: $\quad$ May 8, 2021 

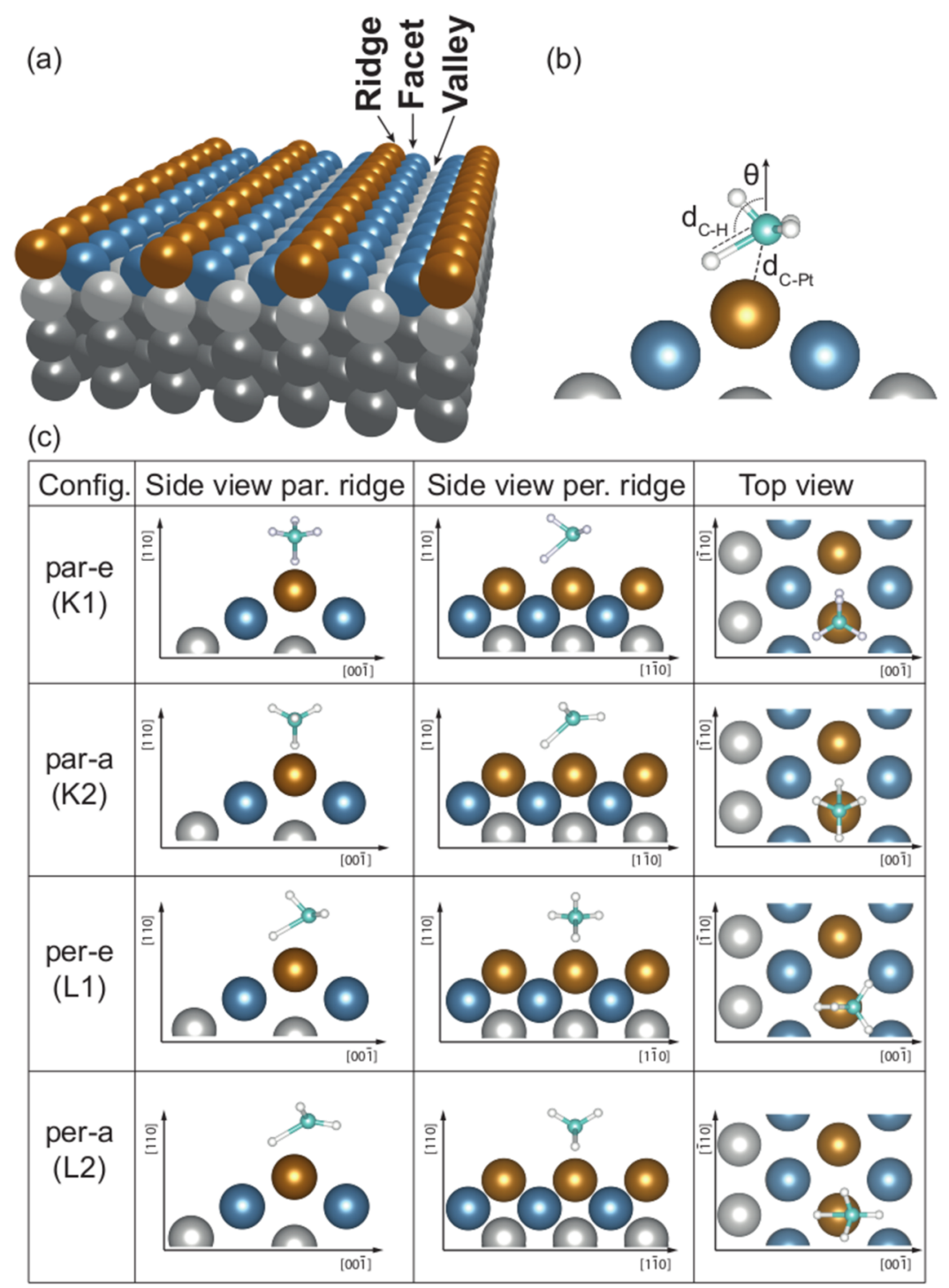

Figure 1. (a) Structure of the $\mathrm{Pt}(110)-(2 \times 1)$ surface and notation used for different types of exposed Pt atoms: ridge, facet, and valley. (b) Coordinates used to define the geometry of TS for methane dissociation on top of a ridge Pt atom. (c) Side views parallel and perpendicular to the row of ridge Pt atoms and top view of the structure of four TS for methane dissociation on top of the ridge Pt atoms: par-e, par-a, per-e, and per-a (see the text).

results of $S_{0}$ obtained through SMB experiments and quasiclassical (QC) density functional molecular dynamics (DFMD) calculations. The comparison (for $T_{\mathrm{s}}=650 \mathrm{~K}$ ) was restricted to high impact energies $\left(E_{\mathrm{i}} \gtrsim 1 \mathrm{eV}\right.$ where $\left.S_{0} \gtrsim 0.05\right)$ to achieve a statistically significant number of reactive DFMD trajectories and still be computationally affordable. ${ }^{24}$ The main aspects of the experimental results were also qualitatively reproduced by the QC DFMD calculations. However, the simulations showed that even for $E_{\mathrm{i}}$ as high as $1 \mathrm{eV}$ : (i) a significant fraction of the incident molecules remain trapped near the surface during at least 1 ps (i.e., much more than the $\sim 0.2$ ps that takes direct dissociation for such relatively fast molecules) and (ii) during that period those molecules can travel up to $25 \AA$ parallel to the surface. ${ }^{24}$ While the DFMD simulations unambiguously showed that for methane $/ \mathrm{Pt}(110)$ $(2 \times 1)$ temporary trapping may occur even for $E_{\mathrm{i}} \sim 1 \mathrm{eV}$ due to the high corrugation of the surface, they could not determine the fate of trapped molecules for trapping times exceeding 1 ps due to prohibitive computational costs. To some extent, this lack of information limited the comparison with experiments and this becomes even more restrictive for lower $E_{\mathrm{i}}$ and/or $T_{\mathrm{s}}$ values. In addition, in view of the observed large lateral displacements of the trapped molecules, the fixed-site approximation involved in the $\mathrm{RPH}$ method used in ref 23 cannot be easily justified a priori.

The results described above give rise to the following questions that motivate the present study. What happens to molecules that remain trapped for more than 1 ps? Will they eventually dissociate and contribute to $S_{0}$ or desorb intact back into the gas phase? Does the fate of such long-interaction-time molecules change significantly when the impact energy and surface temperature decrease? Why is it that the RPH method, which assumes dissociation exclusively on the initial impact, can still provide a qualitatively good description of the measured sticking probabilities even under conditions for which molecular trapping plays a significant role?

To find answers to these questions and to overcome the limitations of DFMD derived from its high computational cost, we use a reactive force field (RFF) specifically developed for this system from DFT results. Since force evaluations are $10^{4}-$ 
$10^{5}$ times faster than in DFMD calculations, this approach allows us to integrate many more trajectories per incidence conditions and for much larger total integration times. Thus, in this work we report a comparison of SMB experiments and QCT results over a wide range of initial conditions: for $0.2 \mathrm{eV}$ $\leq E_{\mathrm{i}} \leq 1.3 \mathrm{eV}$ and $T_{\mathrm{s}}=650$ and $120 \mathrm{~K}$. The present study of methane/Pt(110)-(2×1) sheds light on the fate of the large fraction of long-interaction-time molecules at high impact energies recently reported, and on all of the related questions mentioned above.

\section{METHODOLOGY}

2.1. Theory. 2.1.1. DFT Calculations. DFT calculations have been performed using a plane-wave basis set and the projected augmented-wave method, ${ }^{25}$ as implemented in the Vienna ab initio simulation package (VASP). ${ }^{26-30}$ We have performed spin-restricted calculations using an energy cutoff, $E_{\text {cutoff }}=450 \mathrm{eV}$, and the Perdew-Burke-Ernzerhof (PBE) generalized gradient approximation to describe electronic exchange and correlation. ${ }^{31}$ With these settings, $19 \times 19 \times$ $19 k$-points, and a smearing of $0.1 \mathrm{eV}$, we obtained a lattice constant of bulk Pt equal to $3.97 \AA$, i.e., only $1.3 \%$ bigger than the experimental value of $3.92 \AA^{32}$ The $\mathrm{Pt}(110)-(2 \times 1)$ surface has been modeled within the slab-supercell approach by seven Pt layers and using a $1 \times 3$ supercell using the theoretical lattice constant of bulk $\mathrm{Pt}$ mentioned above. The surface calculations were performed using a $7 \times 7 \times 1 k$-point mesh. We have first optimized the geometry of the clean slab by allowing the relaxation of $\mathrm{Pt}$ atoms in all layers. The obtained lowest-energy clean-surface structure was first kept fixed (rigid surface) for the search of four transition states, TS, reported by Jackson and co-workers (all with the $\mathrm{C}$ atom located on top of a ridge $\mathrm{Pt}$ atom), ${ }^{33}$ using the quasi-Newton algorithm implemented in VASP. To designate these four TS structures, we use $x x x-y$ (with $x x x=$ par, per and $y=\mathrm{a}$, e) to denote a configuration with the breaking $\mathrm{C}-\mathrm{H}$ bond parallel (par) or perpendicular (per) to the ridge, and eclipsed $(e)$ or alternated (a) with respect to the other three $\mathrm{C}-\mathrm{H}$ bonds. The configurations par-e, par-a, per-e, and per-a (respectively, called K1, K2, L1, and L2 in ref 33) are shown in Figure 1c. After having obtained these four TS structures within the rigid surface approximation, we have used them as the starting point of full-geometry optimization allowing the motion of the atoms of both the molecule and the three outermost Pt layers (nonrigid surface).

2.1.2. RFF Parametrization. The DFT-PBE total energies for methane $/ \mathrm{Pt}(110)-(2 \times 1)$ were fitted using an analytical potential $V(\{\mathbf{r}\})$ consisting of a sum of two-body repulsive and attractive potentials $\left(V_{\alpha \beta}^{\mathrm{R}}\left(r_{i j}\right)\right.$ and $V_{\alpha \beta}^{\mathrm{A}}\left(r_{i j}\right)$, respectively), with the latter being modulated by a symmetrized bond-order parameter, $\bar{b}_{i j}^{\alpha \beta}$ that incorporates three-body interactions

$$
\begin{aligned}
V(\{\mathbf{r}\})= & \frac{1}{2} \sum_{\alpha=1}^{n} \sum_{\beta=1}^{n} \sum_{i=1}^{N_{\alpha}} \sum_{\substack{j=1 \\
j \neq i \text { if } \alpha=\beta}}^{N_{\beta}}\left[V_{\alpha \beta}^{\mathrm{R}}\left(r_{i j}^{\alpha \beta}\right)\right. \\
& \left.-\bar{b}_{i j}^{\alpha \beta} V_{\alpha \beta}^{\mathrm{A}}\left(r_{i j}^{\alpha \beta}\right)\right]
\end{aligned}
$$

In eq $1,\{\mathbf{r}\}$ represents the set of position vectors of all of the atoms within the simulation supercell, $n$ is the number of atomic species in the system $(3: \mathrm{Pt}, \mathrm{H}, \mathrm{C}), N_{\alpha}\left(N_{\beta}\right)$ is the number of atoms of species $\alpha(\beta)$, and $r_{i j}^{\alpha \beta}$ is the distance between the $i^{\text {th }}$ atom of species $\alpha$ and the $j^{\text {th }}$ atom of species $\beta$.
The expressions of $V_{\alpha \beta}^{\mathrm{R}}\left(r_{i j}^{\alpha \beta}\right), V_{\alpha \beta}^{\mathrm{A}}\left(r_{i j}^{\alpha \beta}\right)$, and $\bar{b}_{i j}^{\alpha \beta}$ are given in eqs $2-5$ of the Supporting Information, SI. They involve fitting parameters

$$
\mathcal{A}_{\alpha \beta}, \mathcal{B}_{\alpha \beta}, \mathcal{C}_{\alpha \beta}, \mathcal{D}_{\alpha \beta}, \mathcal{F}_{\alpha \beta}, \mathcal{H}_{\alpha \beta \gamma}, \mathcal{L}_{\alpha \beta \gamma}, \mathcal{M}_{\alpha \beta \gamma}, \mathcal{O}_{\alpha \beta \gamma}
$$

whose values have been optimized to minimize the object function

$$
\chi^{2}\left(\mathcal{A}_{\alpha \beta}, \mathcal{B}_{\alpha \beta}, \ldots, O_{\alpha \beta \gamma}\right)=\sum_{j=1}^{N_{\text {conf }}} w_{j}\left(V_{j}^{\mathrm{DFT}}-V_{j}\right)^{2}
$$

using the Levenberg-Marquardt nonlinear weighted leastsquares method. ${ }^{34}$ In eq $2, N_{\text {conf }}$ is the number of configurations included in the database for fitting $\left(N_{\text {conf }} \sim\right.$ $10000)$ and $V_{j}^{\text {DFT }}$ and $V_{j}$ are the total energies of the $j^{\text {th }}$ configuration obtained in DFT calculations and predicted by the interaction potential $V(\{\mathbf{r}\})$ (eq 1 ), respectively. $w_{j}$ is a coefficient associated with the $j^{\text {th }}$ configuration introduced to give weight to low-energy configurations higher than for highenergy ones $\left(w_{j}=1\right.$ if $V_{j}^{\mathrm{DFT}} \leq 1.8 \mathrm{eV}$ and decreases for increasing $V_{j}^{\mathrm{DFT}}$ values, where $V^{\mathrm{DFT}}=0$ corresponds to our reference configuration with the molecule and the surface in the lowest-energy geometry and far from each other).

The initial database for fitting used in this work includes configurations for: (i) the distorted molecule far from the undistorted surface, (ii) the undistorted molecule far from the distorted surface, (iii) the undistorted molecule approaching the surface on different high-symmetry sites and with different orientations (with one, two, and three $\mathrm{H}$ atoms pointing to the surface), and (iv) all of the configurations explored by the geometry-optimization algorithm we have used to search the DFT TS, within both the rigid and nonrigid surface models. Here, the undistorted surface (molecule) refers to the lowestenergy configuration of the surface (molecule) for a large molecule-surface distance. On the other hand, the distorted surface (molecule) refers to the surface (molecule) out of its undistorted configuration. The distorted configurations for both the molecule and the surface were generated by running DFMD calculations starting from initial distorted configurations obtained by displacing some atoms out of the undistorted configuration. More precisely, for the molecule we have performed $\sim 200$-step DFMD calculations for: (i) $\mathrm{CH}_{4}$ initially with $\alpha \times \mathrm{ZPE}(\alpha=1,3 / 2,5 / 2)$ in each vibrational normal mode and zero energy in all of the others (i.e., 12 DFMD calculations), (ii) $\mathrm{CH}_{4}(v=0)$ (i.e., in its ground vibrational state), and (iii) the first and second singly excited states of all of the vibrational normal modes. For the surface, distorted configurations are those visited during: (i) geometry optimization starting from the ideal bulk truncated structure of the $(2 \times 1)$ missing-row reconstructed (110) face of $\mathrm{Pt}$, and from structures of $\mathrm{Pt}(110)-(2 \times 1)$ with various $\mathrm{Pt}$ atoms randomly displaced from their equilibrium positions, and (ii) DFMD calculations for $T_{s}=300,500$, and $1000 \mathrm{~K}$.

The initial database described above has been used to obtain the first version of our RFF, which in turn was used in QCT calculations for various values of $E_{\mathrm{i}}$ up to $1.3 \mathrm{eV}$, and $T_{\mathrm{s}}$ up to $650 \mathrm{~K}$. They were performed to select new configurations, which were then added to the database for refitting. This procedure was repeated many times until convergence of the sticking probabilities was obtained without any doubtful configuration being detected during MD simulations. Additional information on the RFF parametrization method 
employed can be found elsewhere. ${ }^{35}$ The obtained optimum values of the fitting parameters are reported in the SI.

The quality of the fitting of the DFT-PBE total energies and the accuracy of the RFF forces are illustrated in Figure 2a,b,
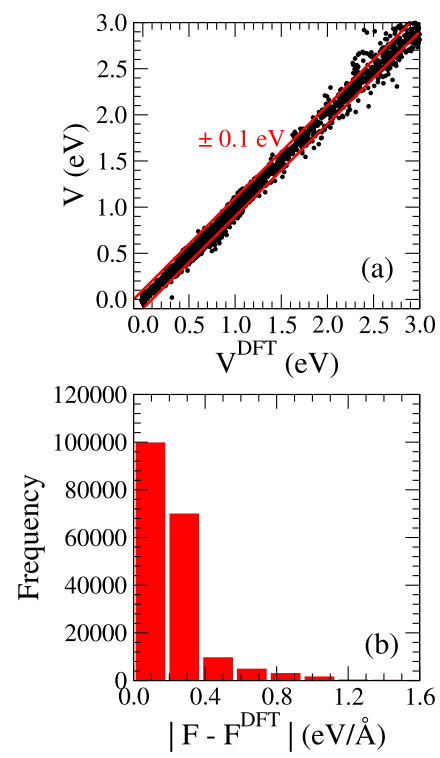

Figure 2. Comparison of results obtained in DFT-PBE calculations and with the interaction potential $V(\{\mathbf{r}\})$ (eq 1) for configurations of methane/ $\operatorname{Pt}(110)-(2 \times 1)$ included in the fitting database (see the text). (a) $V$ vs $V^{\mathrm{DFT}}$ : configurations lying between the two red straight lines have fitting errors smaller than $0.1 \mathrm{eV}$. (b) Distribution of fitting errors affecting the forces acting on the atoms, measured by the modulus of the difference between the RFF and DFT-PBE force, $\mid F-$ $F^{\mathrm{DFT}}$, for configurations with $V^{\mathrm{DFT}} \leq 3 \mathrm{eV}$.

respectively. It is observed that for DFT energies below $\sim 2 \mathrm{eV}$, the fitting errors of the total energy for the great majority of the configurations are smaller than $0.1 \mathrm{eV}$. For configurations with higher DFT energies, the fitting errors increase because of the reduction of the corresponding weighting factors $w_{j}$ in eq 2 . For instance, for all of the configurations in the database with $V^{\mathrm{DFT}} \leq 1.5 \mathrm{eV}$, the computed root-mean-square error (RMSE) is smaller than $0.03 \mathrm{eV}$, and reaches $0.1 \mathrm{eV}$ if all of the configurations with $V^{\mathrm{DFT}} \leq 8 \mathrm{eV}$ are considered. Concerning the forces acting on all of the atoms of the system, for configurations with $V^{\mathrm{DFT}} \leq 3 \mathrm{eV}$ the error of the majority of the forces is smaller than $0.2 \mathrm{eV} / \AA$ and rarely exceeds $0.4 \mathrm{eV} / \AA$ (Figure 2b).

Since the $V_{\alpha \beta}^{\mathrm{R}}\left(r_{i j}^{\alpha \beta}\right)$ and $V_{\alpha \beta}^{\mathrm{A}}\left(r_{i j}^{\alpha \beta}\right)$ potentials (eq 1) are shortranged (they are switched off to zero for $r_{i j}^{\alpha \beta} \geq 3.5 \AA$ ), $V(\{\mathbf{r}\})$ cannot properly describe long-distance van der Waals interactions. In fact, this is consistent with the fact that the PBE-DFT calculations used to build the fitting database do not account for the long-distance molecule-surface attraction due to dispersion forces. Using the specific reaction parameter (SRP32-vdW) method that incorporates van der Waals interactions, Chadwick et al. have studied this long-distance behavior of the molecule-surface interaction potential and have shown that: (i) the physisoprtion well for methane/ $\operatorname{Pt}(110)-(2 \times 1)$ is $\sim 0.28 \mathrm{eV}$ in depth, and (ii) for $1.5 \AA \leq Z_{\mathrm{cm}}$ $\leq 3.5 \AA$ (with $Z_{\mathrm{cm}}=0$ corresponding to the height of ridge $\mathrm{Pt}$ atoms), this long-distance molecule-surface attractive interaction is rather corrugated along the coordinate, $X_{\mathrm{cm}}$, parallel to the surface in the direction perpendicular to the missing $\mathrm{Pt}$ rows. ${ }^{24}$ For $Z_{\mathrm{cm}} \geq 3.5 \AA$, the molecule-surface interaction is attractive but weakly dependent on the surface site and becomes negligible for $Z_{\mathrm{cm}} \gtrsim 7 \AA$. To reproduce these features at long molecule-surface distances, we have added to $V(\{\mathbf{r}\})$ (eq 1), an empirical correction

$$
V^{\mathrm{RFF}}(\{\mathbf{r}\})=V(\{\mathbf{r}\})+V_{\mathrm{vdW}}\left(Z_{\mathrm{cm}}, X_{\mathrm{cm}}\right)
$$

The expression used for $V_{\mathrm{vdW}}\left(Z_{\mathrm{cm}}, X_{\mathrm{cm}}\right)$ can be found in the SI (eqs 6-9). The resulting $Z_{\mathrm{cm}}$-dependence of the vdWcorrected reactive force field, $V^{\mathrm{RFF}}(\{\mathbf{r}\})$, on top of the ridge, facet, and valley Pt atoms are shown in Figure 3 together with

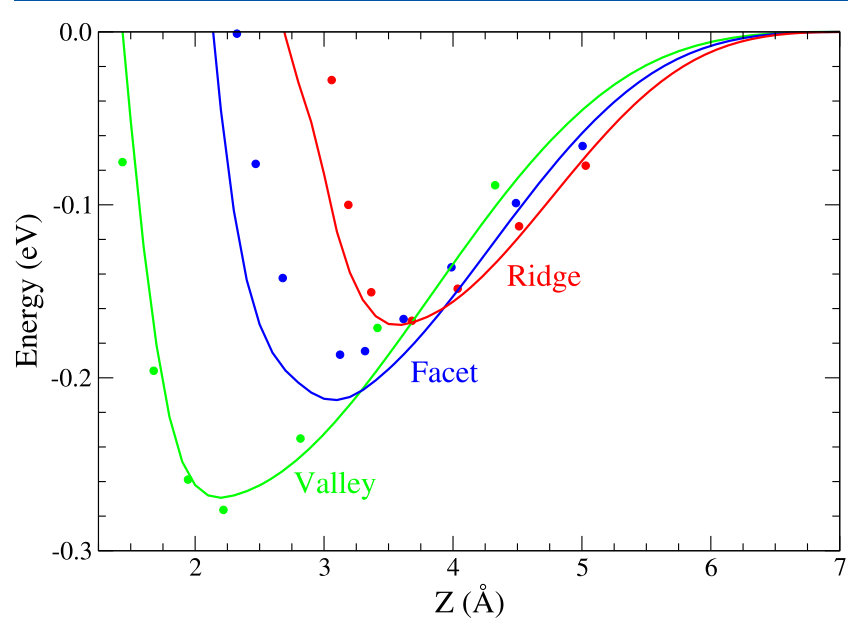

Figure 3. Full lines: $Z_{\mathrm{cm}}$-dependence of $V^{\mathrm{RFF}}$ (eq 3 ) on the ridge (red), facet (blue), and valley (green) $\mathrm{Pt}$ atoms of $\mathrm{Pt}(110)-(2 \times 1)$ ( $Z_{\mathrm{cm}}$ is measured with respect to the position of the ridge Pt atoms). Full circles: SRP32-vdW data extracted from Figure S2 of ref 24.

the SRP32-vdW results extracted from ref 24. It is important to mention that on ridge $\mathrm{Pt}$ atoms, $V_{\mathrm{vdW}}\left(Z_{\mathrm{cm}}, X_{\mathrm{cm}}\right)$ switches off to zero for $Z_{\mathrm{cm}} \leq 2.7 \AA$ (measured with respect to the position of ridge $\mathrm{Pt}$ atoms) and so, $V_{\mathrm{vdW}}$ does not affect either the geometry or the energy of the TS for dissociation obtained with the non-vdW-corrected interaction potential $V(\{\mathbf{r}\})$.

2.1.3. QCT Calculations. To investigate the dynamics of methane dissociative adsorption on $\operatorname{Pt}(110)-(2 \times 1)$ and to compute the corresponding initial reactive sticking probability, $S_{0}$, we have performed QCT calculations. The equations of motion of atomic coordinates and momenta were integrated using the Verlet algorithm. ${ }^{36,37}$ The initial state of the molecule for particular initial vibrational states was selected using a standard procedure following ref 38 . We have considered normal incidence and initial translational energies, $E_{\mathrm{i}}$, between 0.2 and $1.3 \mathrm{eV}$. This choice, certainly motivated by the energy range covered by the new unpublished experimental data reported here, leaves out the low-energy regime where the reactive sticking probability is deactivated by impact energy (i.e., $\left.E_{\mathrm{i}} \lesssim 0.1 \mathrm{eV}\right)^{8,39}$ and becomes much more sensitive to the potential energy surface used in the dynamical calculations, ${ }^{16}$ and to the presence of surface defects such as steps and kinks. ${ }^{16,40}$ We have only considered initially nonrotating methane molecules whose orientation was selected using the standard sampling of Euler angles (see, e.g., ref 41), and the initial impact point was randomly chosen throughout a $1 \times 3$ unit cell (with respect to the reconstructed surface). For the QCT calculations we have kept fixed the positions of the $\mathrm{Pt}$ atoms in the four inner layers of $\mathrm{Pt}(110)-(2 \times 1)$. The initial 
Table 1. DFT Geometry and Energy of Four TS for $\mathrm{CH}_{4} / \mathrm{Pt}(110)-(2 \times 1)$ for the Rigid Surface and Nonrigid Surface Models (See the Text) ${ }^{a}$

\begin{tabular}{|c|c|c|c|c|c|c|c|c|c|}
\hline \multirow[b]{2}{*}{ TS } & \multicolumn{4}{|c|}{ rigid surface } & \multicolumn{5}{|c|}{ nonrigid surface } \\
\hline & $d_{\mathrm{C}-\mathrm{Pt}}(\AA)$ & $d_{\mathrm{C}-\mathrm{H}}(\AA)$ & $\theta\left(^{\circ}\right)$ & $E_{\mathrm{b}}(\mathrm{eV})$ & $d_{\mathrm{C}-\mathrm{Pt}}(\AA)$ & $d_{\mathrm{C}-\mathrm{H}}(\AA)$ & $\theta\left(^{\circ}\right)$ & $E_{\mathrm{b}}(\mathrm{eV})$ & $\Delta_{\mathrm{Pt}}(\AA)$ \\
\hline par-e (K1) & 2.22 & 1.54 & 131 & $0.71(0.70 ; 0.72)$ & 2.21 & 1.52 & 131 & 0.62 & 0.12 \\
\hline par-a (K2) & 2.24 & 1.52 & 133 & $0.74(0.72 ;--)$ & 2.23 & 1.50 & 133 & 0.64 & 0.12 \\
\hline per-e (L1) & 2.20 & 1.62 & 120 & $0.74(0.72 ;--)$ & 2.19 & 1.59 & 118 & 0.62 & 0.14 \\
\hline per-a (L2) & 2.21 & 1.58 & 120 & $0.73(0.71 ; 0.66)$ & 2.20 & 1.55 & 119 & 0.61 & 0.14 \\
\hline
\end{tabular}

${ }^{a} d_{\mathrm{C}-\mathrm{Pt}}, d_{\mathrm{C}-\mathrm{H}}$, and $\theta$ are defined in Figure $1 \mathrm{~b}$, and $\Delta_{\mathrm{Pt}}$ is the outward vertical displacement of the closest Pt atom with respect to its equilibrium position for the clean surface. The $E_{\mathrm{b}}$ values in brackets are those obtained using the PBE functional by Jackson and co-workers ${ }^{23}$ (left) and the SRP32-vdW method by Kroes and co-workers ${ }^{24}$ (right).

coordinates and momenta of the $\mathrm{Pt}$ atoms in the three outermost layers were selected from a large set of lattice configurations produced previously in a NVT-MD simulation of the clean surface. The $\mathrm{Pt}$ atoms in the third layer have been put in contact with a Berendsen thermostat. Trajectories started with the molecular center of mass $Z_{\mathrm{cm}}=8 \AA$ above the height of the ridge $\mathrm{Pt}$ atoms. Whenever a molecule comes back to $Z_{\mathrm{cm}}=8 \AA$ from the surface (one of the molecular bonds reaches the $1.875 \AA$ with the projection of the velocities of both active atoms along their internuclear axis tending to separate them from each other), the molecule is considered reflected (dissociated). At that moment the integration of the corresponding trajectory is stopped. If none of these events takes place, at a maximum integration time, $t_{\max }$ we stop the trajectory and considered that the molecule is trapped intact near the surface. To investigate the fate of molecules after a long trapping time and to minimize the number of trajectories that have neither led to desorption nor to dissociation, we have increased the integration time to $t_{\max }=1000$ ps (see Section 3.4). The total number of trajectories integrated for each initial condition was chosen to be between $2 \times 10^{4}$ and $4 \times 10^{5}$ depending on the value of $S_{0}$, to obtain results as well statistically converged as possible.

2.2. Experiments. The molecular beam-surface science apparatus used in this study has been described in detail previously. $^{42}$ Briefly, the apparatus consists of a 3-fold differentially pumped molecular beam source coupled to an ultrahigh vacuum (UHV) surface science chamber with a base pressure of $5 \times 10^{-11}$ mbar. In the source chamber, a continuous molecular beam was formed by skimming a jet expansion produced by expanding a gas mixture of $1-3$ bar stagnation pressure through a stainless steel nozzle. Three different gas mixtures were used: $3 \% \mathrm{CH}_{4}$ in $\mathrm{He}, 1 \% \mathrm{CH}_{4}$ in $\mathrm{H}_{2}$, and $1.7 \% \mathrm{CHD}_{3}$ in $\mathrm{H}_{2}$. The translational energy of the molecular beam was controlled by changing the nozzle temperature in the range of $300-800 \mathrm{~K}$. The speed distribution of the molecular beam was measured by a timeof-flight method using a chopper wheel in combination with an on-axis quadrupole mass spectrometer. ${ }^{43}$ The molecular beam collided in the UHV chamber with a circular $10 \mathrm{~mm}$ diameter $\operatorname{Pt}(110)-(2 \times 1)$ surface at normal incidence. The single-crystal sample was obtained from Surface Preparation Labs (Zaandam, the Netherlands) and was cut within $0.1^{\circ}$ of the (110) plane. The crystal disk was mounted on a liquid nitrogen cryostat using tungsten wires, which could be heated resistively to control the surface temperature in the range of 90-1200 K. Surface cleaning before each reactivity measurement was performed by $\mathrm{Ar}^{+}$sputtering and/or by exposing the surface to $5 \times 10^{-8}$ mbar of $\mathrm{O}_{2}$ at a surface temperature of $700 \mathrm{~K}$ for 5 min followed by annealing at $T_{s}=1100 \mathrm{~K}$ for $2 \mathrm{~min}$. The surface cleanliness was verified using Auger electron spectroscopy (AES), confirming that no detectable trace of carbon $(<1 \% \mathrm{ML})$ was on the surface. Two different techniques were used to measure the initial sticking coefficients $\left(S_{0}\right)$ of methane on $\mathrm{Pt}(110)-(2 \times 1)$. At low surface temperature, $T_{\mathrm{s}}=120 \mathrm{~K}$, we used reflection absorption infrared spectroscopy (RAIRS) to detect and quantify the coverage of the nascent methyl products formed by methane dissociation. We previously reported $^{44,45}$ the surface site selective RAIRS detection of methane dissociation on the steps and terrace sites of a $\operatorname{Pt}(211)$ surface. On $\operatorname{Pt}(110)-(2 \times 1)$, we detect methane dissociation only on the ridge sites in agreement with early studies from our laboratory. ${ }^{46}$ Initial sticking coefficients of methane are obtained from the initial slope of the uptake curve obtained from the RAIRS signal calibrated in terms of surface coverage. A more detailed description of this procedure can be found elsewhere. ${ }^{44,45}$ At surface temperatures above $180 \mathrm{~K}$, the nascent methyl product species are unstable and quickly dehydrogenate leading to $\mathrm{C}$ (ads) and hydrogen desorption. Therefore, we used the molecular beam reflectivity method developed by King and Wells ${ }^{24,47}$ to measure the initial sticking coefficients at $T_{\mathrm{s}}=650 \mathrm{~K}$.

\section{RESULTS AND DISCUSSIONS}

3.1. DFT Results. The geometry and energy of the four transition states (TS) obtained by DFT calculations within the rigid and the nonrigid surface approximation are summarized in Table 1 . All four TS have very similar energies: $0.72 \pm 0.02$ and $0.62 \pm 0.02 \mathrm{eV}$ for the rigid- and nonrigid-surface models, respectively. The smallest $\mathrm{C}-\mathrm{Pt}$ distance, $d_{\mathrm{C}-\mathrm{Pt}}$, the $\mathrm{C}-\mathrm{H}$ distance for the breaking bond, $d_{\mathrm{C}-\mathrm{H}}$, and the polar angle between the $\mathrm{C}-\mathrm{H}$ breaking bond and the surface normal, $\theta$ (see Figure 1b) are similar for the four TS, though per configurations are characterized by values of $d_{\mathrm{C}-\mathrm{H}} \sim 0.05 \AA$ larger $\left(\theta \sim 12^{\circ}\right.$ smaller $)$ than the par ones. All of the geometric and energetic properties of the TS we have obtained within the rigid-surface model agree very well with those reported previously by Jackson and co-workers ${ }^{23,33}$ who used DFT settings very similar to the ones used here. The energies we have obtained for the $\mathrm{K} 1$ (L2) TS is $0.01 \mathrm{eV}$ smaller $(0.07 \mathrm{eV}$ larger) than the one reported by Chadwick et al. who used the specific reaction parameter (SRP32-vdW) approach to model the methane $/ \operatorname{Pt}(110)-(2 \times 1)$ system. ${ }^{24}$ Within the nonrigid surface approximation, the energy barriers we have obtained for par and per configurations are, respectively, 0.09 and 0.12 $\mathrm{eV}$ smaller than for the rigid surface. As pointed out in ref 23, this energy decrease is due to the shift up of the closest $\mathrm{Pt}$ atom by 0.12 and $0.14 \AA$, respectively (see Table 1 ). This energy-barrier decrease $(0.09-0.12 \mathrm{eV})$, when surface relaxation is allowed, is consistent with the $\sim 0.07 \mathrm{eV}$ decrease 
reported in ref 33 (for the $\mathrm{K} 1$ and $\mathrm{L} 1 \mathrm{TS}$ ) only for the ridge $\mathrm{Pt}$ atom closest to the molecule shifted up by $0.1 \AA$.

3.2. RFF Accuracy. In Table 2, we compare the interlayer distance between the three outermost layers (the ones allowed

Table 2. Difference between the Bulk and the Relaxed Geometry of $\operatorname{Pt}(110)-(2 \times 1)^{a}$

\begin{tabular}{|c|c|c|c|c|}
\hline method & $\Delta d_{12}(\%)$ & $\Delta d_{23}(\%)$ & $\Delta d_{34}(\%)$ & $b_{3}(\AA)$ \\
\hline DFT (present work) & -19.5 & -0.3 & +1.9 & 0.35 \\
\hline DFT (ref 24) & -18.5 & -0.2 & +1.1 & 0.35 \\
\hline RFF & -18.1 & -2.4 & +1.1 & 0.37 \\
\hline exp. LEED $^{48}$ & -17.4 & +1.1 & +0.4 & 0.17 \\
\hline exp. LEED $^{49}$ & -18.4 & -12.6 & -8.7 & 0.32 \\
\hline exp. MEIS ${ }^{50}$ & -16 & +4 & & 0.10 \\
\hline exp. X-ray diffraction ${ }^{51}$ & -19.5 & -7.9 & & \\
\hline
\end{tabular}

${ }^{a} d_{i j}$ represents the distance between the layers $i$ and $j$, with $i=1$ corresponding to the outermost surface layer, and $b_{3}$ represents the buckling in the third layer (see Figure 4).

to relax) obtained in the geometry optimization of the clean surface geometry through DFT and RFF-based calculations (see Figure 4). It is observed that the DFT values are well reproduced by our RFF, discrepancies being not larger than $0.02 \AA$.

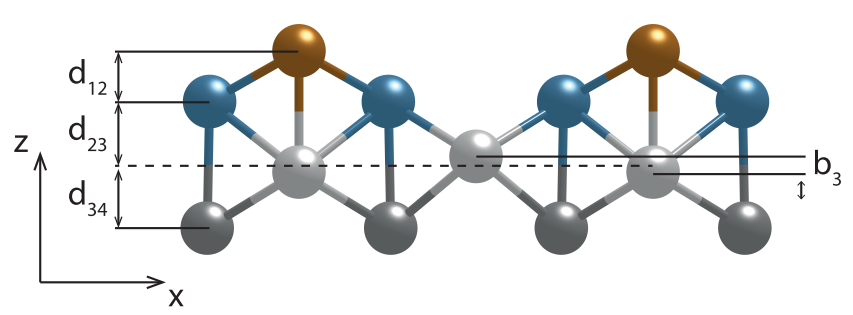

Figure 4. Side view of $\operatorname{Pt}(110)-(2 \times 1)$ with the definition of the structural parameters $\mathrm{d}_{i j}, i=1,2,3 ; j=i+1$, and $b_{3}$ reported in Table 2.

In Table 3, we compare the frequencies of the vibrational modes of $\mathrm{CH}_{4}$ and $\mathrm{CHD}_{3}$ in a vacuum obtained through DFT and RFF-based calculations (within the harmonic approximation) with experimental data extracted from ref 52. For all of the normal modes, the discrepancies between both theoretical results and between them and experiments are smaller than $100 \mathrm{~cm}^{-1}$. The experimental ordering of the

Table 3. Normal-Mode Frequencies, $\nu$, and Degenerancies, $\lambda$, of $\mathrm{CH}_{4}$ and $\mathrm{CHD}_{3}$ Obtained through DFT and RFFBased Calculations within the Harmonic Approximation and Experimental Data Extracted from Ref 52

$\begin{array}{ccccc} & \lambda & \nu^{\mathrm{RFF}}\left(\mathrm{cm}^{-1}\right) & \nu^{\mathrm{DFT}}\left(\mathrm{cm}^{-1}\right) & \nu^{\text {exp }}\left(\mathrm{cm}^{-1}\right) \\ \mathrm{CH}_{4} & 3 & 3109 & 3092 & 3019.49 \\ & 1 & 2966 & 2975 & 2916.49 \\ & 2 & 1516 & 1514 & 1533.34 \\ \mathrm{CHD}_{3} & 3 & 1328 & 1288 & 1310.76 \\ & 1 & 3078 & 3065 & 2992.75 \\ & 2 & 2302 & 2286 & 2250.83 \\ & 1 & 2143 & 2145 & 2142.58 \\ & 2 & 1285 & 1271 & 1292.50 \\ & 2 & 1033 & 1012 & 1035.52 \\ & 1 & 1011 & 980 & 1004.55\end{array}$

frequency values is also well reproduced. These results support the use of the RFF developed here to describe the dynamics of $\mathrm{CH}_{4}$ and $\mathrm{CHD}_{3}$ initially in the ground vibrational state and in vibrationally excited states.

The TS obtained using the resulting RFF within the rigid and nonrigid surface approximations are shown in Table 4. Within the rigid surface approximation, the obtained activation energies and geometries of the four considered TS are similar to the DFT ones. For instance, the discrepancies between DFT and RFF energies, $\mathrm{C}-\mathrm{Pt}$ distances, $\mathrm{C}-\mathrm{H}$ distances, and $\theta$ angles are never larger than $0.04 \mathrm{eV}, 0.06 \AA, 0.12 \AA$, and $6^{\circ}$ respectively. Though, some of these discrepancies are in principle not negligible (e.g., the $0.12 \AA$ difference in $d_{\mathrm{C}-\mathrm{H}}$ for the par-a configuration), in general the agreement is good and in particular, the RFF reproduces the $\theta$ values for per configurations $\sim 10^{\circ}$ smaller than for par ones found in DFT calculations. For the nonrigid surface approximation, the agreement between the RFF and DFT results deteriorates, with discrepancies for energy barriers varying between 0.07 and 0.18 $\mathrm{eV}$. The RFF energy barriers are all lower than the DFT ones. This seems to be connected with too large upshifts of the ridge $\mathrm{Pt}$ atom closest to the molecule in the TS configuration. The attempts we have made to further improve the description of these energy barriers by increasing the weight of configurations near the DFT TS for the nonrigid surface were unsuccessful: in doing that, the quality of the fitting for many other configurations in the database deteriorates significantly. Still, due the predicted weak dynamic coupling between molecular and lattice degrees of freedom because of the mass mismatch, it is expected that the effect of these errors will not be dramatic at least for low temperatures for which thermally activated lattice distortions are small.

3.3. Comparison of QC RFF and DFMD Results. In contrast with DFMD calculations that employ forces computed directly using DFT, the results of any dynamical method using a PES built by interpolation or fitting of DFT data can be affected by the (unavoidable) errors of any PES-parametrization method. Therefore, comparing PES-based-dynamics and DFMD results (obviously based on similar DFT calculations) is important to check the reliability of the PES. ${ }^{53}$ For this reason, here we compare the results obtained with the PBE functional used in this study and the DFMD ones reported by Chadwick et al. using the reaction parameter (SRP32-vdW) approach for the $\mathrm{CHD}_{3} / \mathrm{Pt}(110)-(2 \times 1)$ system. ${ }^{24}$ Despite the different functionals, both studies predict similar geometries and energies (differing by only $50-70 \mathrm{meV}$ ) for the four transition states for methane dissociation on the ridge $\mathrm{Pt}$ atoms. In addition, our RFF has been designed to properly account for the long-distance behavior of the molecule-surface interaction obtained within the SRP approach, as was explained above.

In Figure 5, we compare $S_{0}$ (panel a) and the fraction of dissociation events taking place on ridge $\mathrm{Pt}$ atoms (panel $\mathrm{b}$ ), obtained by our RFF-based QCT calculations and by DFMD. Here, we consider a dissociation event to take place on a ridge $\mathrm{Pt}$ atom when it is closest to the methane $\mathrm{C}$ atom at the time of dissociation where we stop the integration of the corresponding trajectory. For a direct comparison, all of the results presented in Figure 5 have been obtained with the same total integration time, $t_{\max }=1 \mathrm{ps}$, as used in the DFMD calculations. ${ }^{24}$ In general, our RFF-based $S_{0}$ values are in good qualitative agreement with the DFMD ones. Our $S_{0}$ values are 25-45\% lower than the DFMD ones of ref 24 which is not 
Table 4. Same as Table 1 but Results Obtained with the RFF

\begin{tabular}{|c|c|c|c|c|c|c|c|c|c|}
\hline \multirow[b]{2}{*}{ TS } & \multicolumn{4}{|c|}{ rigid surface } & \multicolumn{5}{|c|}{ nonrigid surface } \\
\hline & $d_{\mathrm{C}-\mathrm{Pt}}(\AA)$ & $d_{\mathrm{C}-\mathrm{H}}(\AA)$ & $\theta\left(^{\circ}\right)$ & $E_{\mathrm{b}}(\mathrm{eV})$ & $d_{\mathrm{C}-\mathrm{Pt}}(\AA)$ & $d_{\mathrm{C}-\mathrm{H}}(\AA)$ & $\theta\left(^{\circ}\right)$ & $E_{\mathrm{b}}(\mathrm{eV})$ & $\Delta_{\mathrm{Pt}}(\AA)$ \\
\hline par-e (K1) & 2.19 & 1.55 & 132 & 0.72 & 2.21 & 1.44 & 141 & 0.53 & 0.19 \\
\hline par-a (K2) & 2.19 & 1.55 & 130 & 0.77 & 2.19 & 1.51 & 134 & 0.59 & 0.19 \\
\hline per-e (L1) & 2.18 & 1.76 & 124 & 0.77 & 2.15 & 1.69 & 124 & 0.57 & 0.24 \\
\hline per-a (L2) & 2.18 & 1.80 & 122 & 0.75 & 2.16 & 1.68 & 123 & 0.56 & 0.22 \\
\hline
\end{tabular}

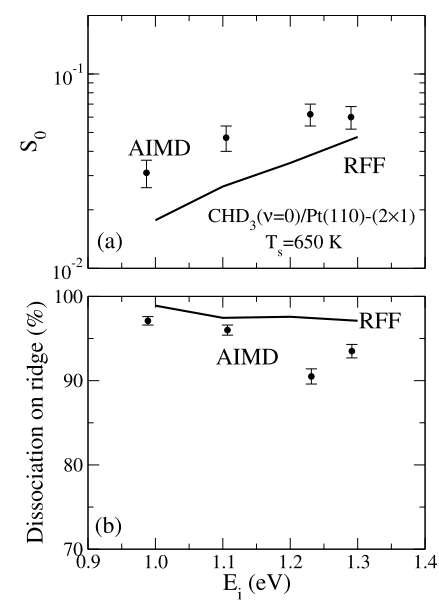

Figure 5. Dynamics results for $\mathrm{CHD}_{3}(v=0) / \mathrm{Pt}(110)-(2 \times 1)$ at $T_{\mathrm{s}}=$ $650 \mathrm{~K}$, obtained using the RFF developed in this work, and through DFMD calculations (results taken from ref 24), in both cases with a total integration time of, $t_{\max }=1$ ps. (a) Initial reactive sticking probability, $S_{0}$ and (b) fraction of dissociation events taking place on ridge $\mathrm{Pt}$ atoms.

unexpected since in our rigid surface some energy barriers are higher than the ones obtained in the latter work by $\sim 0.07 \mathrm{eV}$ (e.g., 0.73 vs $0.66 \mathrm{eV}$ for the L2 TS, as shown in Table 1). However, these discrepancies might also be due to the fact that in contrast with ref 24 , we have not considered the velocityand vibrational-state-distribution of the molecules in SMB experiments. Both RFF-based dynamics and DFMD calculations predict a high fraction of dissociation events taking place on ridge $\mathrm{Pt}$ atoms for $1.0 \mathrm{eV} \lesssim E_{\mathrm{i}} \lesssim 1.3 \mathrm{eV}$ : i.e., higher than $90 \%$ and slightly decreasing for increasing $E_{\mathrm{i}}$. Also in agreement with ref 24 , we have obtained that in this energy range, $\sim 1-5 \%$ of the impinging $\mathrm{CHD}_{3}(\mathrm{v}=0)$ molecules remain trapped near the surface after 1 ps. This fraction of trapped molecules, $F_{\text {trapp }}^{1}$, increases for decreasing energies, as shown in Figure 6 (red line). Although $F_{\text {trapp }}^{1 \text { ps }}$ is relatively small, it is not negligible compared with the reactive sticking

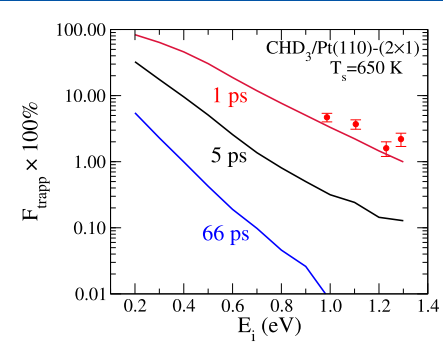

Figure 6. Percentage of molecules that remain trapped (intact) near the surface after 1 ps (red), 5 ps (black), and 66 ps (blue). Lines: RFF-based QCT results and full circles: DFMD results extracted from ref 24. probability. Thus, our results show that $t_{\max }=1$ ps is not long enough to compute dissociative sticking probabilities of $\mathrm{CHD}_{3}(\mathrm{v}=0)$ on $\operatorname{Pt}(110)-(2 \times 1)$ for energies $E_{\mathrm{i}} \leq 1 \mathrm{eV}$, in agreement with DFMD calculations. ${ }^{24}$

Due to the much lower computational cost of our RFFbased approach, we can use $t_{\max }$ values much larger than 1 ps. Then, we have evaluated the fraction of trapped molecules at 5 ps, $F_{\text {trapp }}^{5 \mathrm{ps}}$ and at $66 \mathrm{ps}, F_{\text {trapp }}^{66 \mathrm{ps}}$, which are also shown in Figure 6. The value of $t_{\max }=66 \mathrm{ps}$ was chosen because it is the estimated trapping time of physisorbed $\mathrm{CHD}_{3}$ on $\mathrm{Pt}(110)-(2 \times 1)$ at $T_{\mathrm{s}}=$ $650 \mathrm{~K}^{24}$

Figure 6 shows that in general, $F_{\text {trapp }}$ increases when $E_{\mathrm{i}}$ decreases. For example, $F_{\text {trapp }}^{1 \text { ps }} \times 100 \%$ exceeds $50 \%$ for $E_{\mathrm{i}}<0.4$ $\mathrm{eV}$. As expected, $F_{\text {trapp }}$ decreases strongly when the total integration time $t_{\max }$ is increased. However, at low $E_{\mathrm{i}}$ values, $F_{\text {trapp }}$ is not negligible even for total interaction times as long as 66 ps. For instance, for $E_{\mathrm{i}}=0.2 \mathrm{eV}, T_{\mathrm{s}}=650 \mathrm{~K}$, and $F_{\text {trapp }}^{66 \mathrm{ps}}=$ 0.05: i.e., a value much higher than the reactive sticking probability. ${ }^{8}$ This highlights the importance of long integration times for the methane $/ \mathrm{Pt}(110)-(2 \times 1)$ system. To investigate the fate of the long-time trapped molecules, in our calculations we have used $t_{\max }=1000 \mathrm{ps}$. In the next section, we make use of such long-term simulations and also of the possibility to integrate up to $\sim 10^{5}$ trajectories per initial condition, to perform an extended comparison of QCT results with available and unpublished SMB experimental data, for both $\mathrm{CHD}_{3}$ and $\mathrm{CH}_{4}$, in a wide range of impact energies $\left(0.2 \mathrm{eV} \leq E_{\mathrm{i}} \leq 1.3 \mathrm{eV}\right)$ and surface temperatures $\left(T_{\mathrm{s}}=650\right.$ and $\left.120 \mathrm{~K}\right)$, not accessible for DFMD due to its higher computational cost.

3.4. Comparison of RFF-Based QCT Results with Experiments. 3.4.1. $\mathrm{CHD}_{3}$. In Figure $7 \mathrm{a}$, we compare RFFbased QCT results of $S_{0}\left(E_{\mathrm{i}}\right)$ for $\mathrm{CHD}_{3}(v=0) / \mathrm{Pt}(110)-(2 \times 1)$ with experimental data for $T_{s}=650 \mathrm{~K}$ extracted from ref 24 . In this case, we have found that $t_{\max }=1000 \mathrm{ps}$ is large enough to determine the fate of all molecules even for the smallest $E_{\mathrm{i}}$ values under consideration. The $E_{\mathrm{i}}$-dependence of the SMB experimental data is qualitatively reproduced by theory but the simulation results underestimate the measured data. It must be noted that the experimental data are for molecules with a thermal vibrational distribution corresponding to the nozzle temperature, whereas the calculations are for $\mathrm{CHD}_{3}(\mathrm{v}=0)$. This may explain at least in part why theory underestimates the experimental results in particular for the highest energies considered for which the nozzle temperature and the fraction of vibrationally excited molecules in the beam are the highest. However, the size of the underestimation observed in Figure 7 a does not decrease when $E_{\mathrm{i}}$ decreases, which suggests the existence of other possible source of errors. A $0.3 \mathrm{eV}$ shift to lower $E_{\mathrm{i}}$ of the theoretical curve (the thick line in Figure $7 \mathrm{a}$ ) is needed to overlap the calculated results with the experimental values, which suggests that the average energy barrier for dissociation predicted by our RFF might be too high by $0.3 \mathrm{eV}$. Such an error might be attributed to a too high activation energy obtained with our modeling of the methane/ $\mathrm{Pt}(110)$ - 

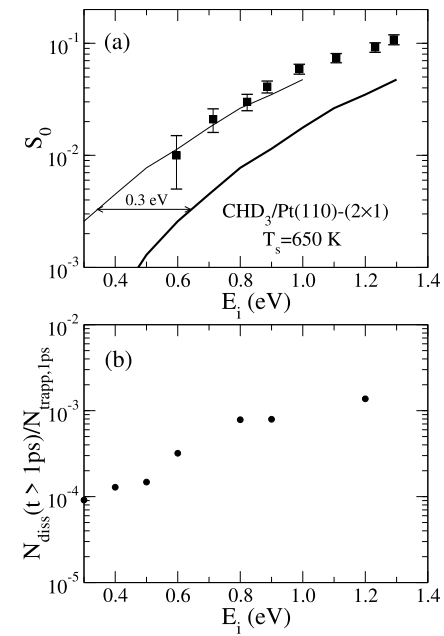

Figure 7. (a) Dissociative sticking probability, $S_{0}$, for $\mathrm{CHD}_{3} / \mathrm{Pt}(110)$ $(2 \times 1)$ at $T_{s}=650 \mathrm{~K}$ as a function of the incident translational energy $E_{\mathrm{i}}$. Squares: experimental data taken from ref 24; full thick line: QCT results for $\mathrm{CHD}_{3}(\mathrm{v}=0)$; and full thin line: QCT results for $\mathrm{CHD}_{3}(v$ $=0$ ) shifted to lower $E_{\mathrm{i}}$ by $0.3 \mathrm{eV}$ (see the text). (b) Fraction of molecules that finally dissociate, after interacting more than 1 ps with the surface.

$(2 \times 1)$ system, in particular the choice of a slab of seven $\mathrm{Pt}$ layers to represent the surface, and the use of the PBE XC functional. Still, in the light of the analysis performed in ref 24, these errors should be not much larger than $0.15 \mathrm{eV}$. Though, the test calculations performed to gauge the quality of our RFF do not point to the fitting procedure as an extra cause of significant errors, this possibility cannot be completely ruled out.

As mentioned above, the QCT-DFMD sticking probabilities presented in ref 24 also underestimate the experiments by a factor of $\sim 2$, and it was argued that dissociation events after interaction times larger than 1 ps might be the reason for such disagreement. In fact, the authors showed that if most of the molecules that remain trapped near the surface at $t=1$ ps eventually dissociate, their DFMD results would be much closer to experiments. However, Figure $7 \mathrm{~b}$ shows that the fraction of $\mathrm{CHD}_{3}$ trapped near the surface at $t=1 \mathrm{ps}$, that eventually dissociate, $N_{\text {diss }}(t>1 \mathrm{ps}) / N_{\text {trapp }}(t=1 \mathrm{ps})$, is smaller than $2 \times 10^{-3}$. If as expected, a similar result would be obtained in DFMD calculations, dissociation events at interaction times larger than 1 ps cannot explain the discrepancy with experiments found in ref 24. In fact, our findings are encouraging for the use of DFMD for methane/ $\operatorname{Pt}(110)-(2 \times 1)$ because they suggest that it is not necessary to run long DFMD trajectories to evaluate the dissociative adsorption probability simply because for $E_{\mathrm{i}}>0.3 \mathrm{eV}, \mathrm{CHD}_{3}(v$ $=0$ ) molecules trapped more than 1 ps do not contribute significantly to $S_{0}$, at least for $T_{\mathrm{s}}=650 \mathrm{~K}$. While the large methane dissociation barrier calculated to be $\sim 0.6-0.7 \mathrm{eV}$ for a static surface with all $\mathrm{Pt}$ atoms fixed at their equilibrium positions (rigid surface approximation) ${ }^{24}$ may seem to exclude the dissociation of trapped molecules, it also neglects any effect due to thermally induced corrugation. One could imagine that the trapped molecules on a hot metal surface at $T_{\mathrm{s}} \sim 650 \mathrm{~K}$ have a finite probability of finding a metal atom which is thermally displaced out of the surface plane and which presents a significantly lower dissociation barrier. ${ }^{54-56}$ However, our present QCT calculations show that the probability of such events is negligible for $\mathrm{CHD}_{3} / \mathrm{Pt}(110)-(2 \times 1)$ at the surface temperatures considered in experiments. ${ }^{24,57}$

3.4.2. $\mathrm{CH}_{4}$. In Figure 8, we compare RFF-based QCT results of $S_{0}$ with new molecular beam experimental data for $\mathrm{CH}_{4} /$

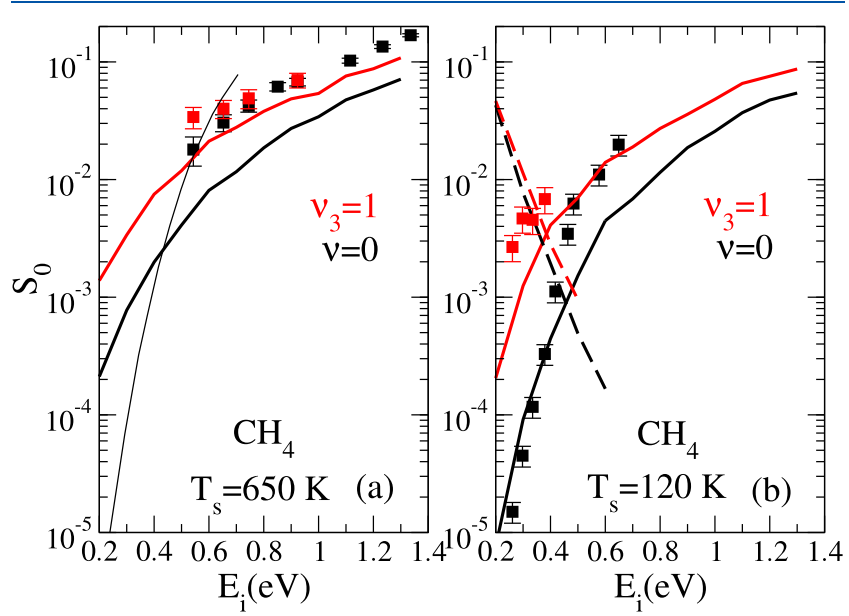

Figure 8. Dissociative sticking probability, $S_{0}$, of $\mathrm{CH}_{4}$ on $\mathrm{Pt}(110)$ $(2 \times 1)$ at (a) $T_{\mathrm{s}}=650 \mathrm{~K}$ and (b) $T_{\mathrm{s}}=120 \mathrm{~K}$. Full thick lines: theoretical results (present work) for $\mathrm{CH}_{4}(v=0)$ (black) and $\mathrm{CH}_{4}\left(\nu_{3}\right.$ $=1)$ (red). Full squares: SMB experiments (present work) for a beam of $\mathrm{CH}_{4}$ molecules with a thermal distribution of initial vibrational states (black) and in the $\nu_{3}=1$ initial state (red). In (a) the full thin black line represents $\mathrm{RPH}$ results for $\mathrm{CH}_{4}(v=0)$ and $T_{\mathrm{s}}=600 \mathrm{~K}$ extracted from ref $23 \mathrm{In}$ (b) the black (red) dashed lines represent the fraction of $\mathrm{CH}_{4}$ molecules in the $v=0\left(\nu_{3}=1\right)$ initial state that remain trapped intact near the surface at $t_{\max }=1000$ ps (see the text).

$\operatorname{Pt}(110)-(2 \times 1)$ at $T_{s}=650 \mathrm{~K}$ (panel a) and $T_{s}=120 \mathrm{~K}$ (panel b). All of the reported QCT results have been obtained using $t_{\max }=1000$ ps. As in the case of $\mathrm{CHD}_{3}$, the theoretical results show an $E_{\mathrm{i}}$-dependence of $S_{0}$ similar to the experimental one but underestimate the measured values except for $\mathrm{v}=0, T_{\mathrm{s}}=$ $120 \mathrm{~K}$, and $E_{\mathrm{i}} \lesssim 0.4 \mathrm{eV}$ (see below). As expected, the $S_{0}$ values for $\mathrm{CH}_{4}\left(\nu_{3}=1\right)$ are higher than those for $\mathrm{CH}_{4}(v=0)$ but both sticking curves present similar $E_{\mathrm{i}}$-dependencies. For comparison, in Figure $8 \mathrm{a}$ we have also included $\mathrm{RPH}$ results for $v=0$ and $T_{\mathrm{s}}=600 \mathrm{~K}$ extracted from ref 23 , which present a stronger $E_{\mathrm{i}}$-dependence (in spite of using the same PBE exchangecorrelation functional as used here to describe the moleculesurface PES), and they reproduce well the experimental data for $E_{\mathrm{i}}=0.55 \mathrm{eV}$ but tend to overestimate the measurements for higher impact energies.

It is important to mention that whereas for the $T_{\mathrm{s}}=650 \mathrm{~K}$ setting, $t_{\max }=1000 \mathrm{ps}$ is sufficient for all of the molecules to either dissociate or reflect to vacuum (for both $v=0$ and $\nu_{3}=1$ initial states and all impact energies), this is not true for $T_{\mathrm{s}}=$ $120 \mathrm{~K}$ at low impact energies: $E_{\mathrm{i}}<0.6 \mathrm{eV}(0.5 \mathrm{eV})$ for $\mathrm{CH}_{4}(v=$ 0) $\left(\mathrm{CH}_{4}\left(\nu_{3}=1\right)\right)$. The fact that even after $t_{\max }=1000 \mathrm{ps}$, at $T_{\mathrm{s}}$ $=120 \mathrm{~K}$ a significant fraction of low-energy molecules remain trapped near the surface (dashed lines in Figure $8 \mathrm{~b}$ ) is not totally surprising because in general, the trapping probability and the lifetime of trapped molecules increase when both $E_{\mathrm{i}}$ and $T_{s}$ decrease. For instance, for methane/ $\operatorname{Pt}(110)-(2 \times 1)$ if molecules thermalize in the $\sim 0.28 \mathrm{eV}$ deep physisorption well (see Figure 3), the estimated lifetime of the physisorbed state $\tau\left(T_{\mathrm{s}}\right)=10^{-13} \mathrm{~s} \times \exp \left(0.27 \mathrm{eV} / K_{\mathrm{B}} T_{\mathrm{s}}\right)$ is $\sim 15$ ps for $T_{\mathrm{s}}=650 \mathrm{~K}$ but $\sim 0.06 \mathrm{~s}$ for $T_{\mathrm{s}}=120 \mathrm{~K}$. Unfortunately, a realistic theoretical description of the time evolution of molecules for 
very long-interaction times is not trivial due not only to a prohibitive computational cost but also to other more fundamental reasons. For very long-interaction times and very small sticking probabilities, classical artifacts like ZPE violation might provoke non-negligible errors. ${ }^{58}$ In addition, the expected large lateral displacements of molecules during their long trapping times increase the role played by surface defects that cannot be avoided in the experiments but are not accounted for in our model. ${ }^{16,40}$ Finally, the description of energy dissipation to the surface through electron-hole pair excitations ${ }^{59}$ might also be necessary for a better description of such long-interaction times. Still, it is interesting to explore if our $\mathrm{MD}$ simulations can provide some clue on the most probable fate of the trajectories characterized by interaction times $>1000$ ps, at least for the ideal case of a perfect (defectfree) surface. With this aim, for $T_{\mathrm{s}}=120 \mathrm{~K}$ we have analyzed the distribution of dissociation and reflection/desorption times of $\mathrm{CH}_{4}(v=0)$ with $E_{\mathrm{i}}=0.4 \mathrm{eV}$ and $\mathrm{CH}_{4}\left(\nu_{3}=1\right)$ with $E_{\mathrm{i}}=0.2$ $\mathrm{eV}$. The results are shown in Figure 9a,b, respectively, where

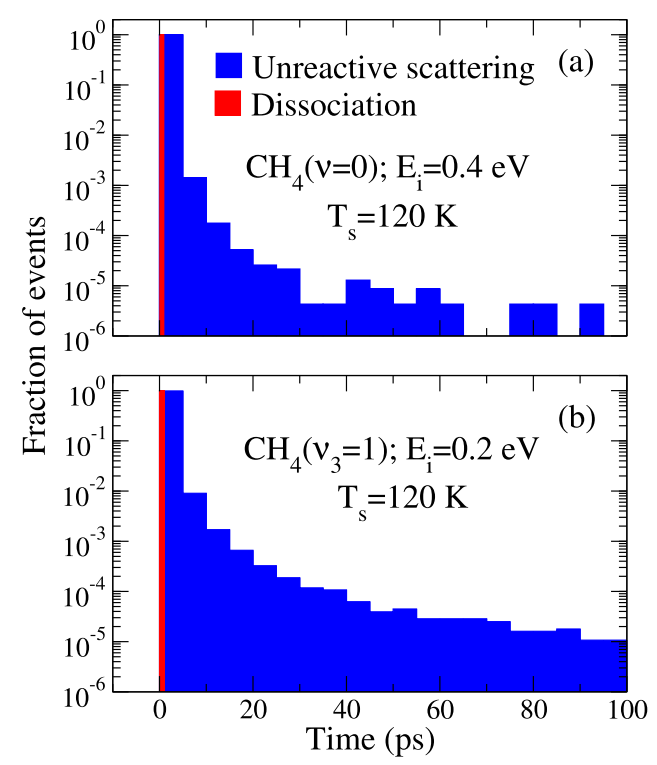

Figure 9. (a) Distributions of reflection (red) and dissociative sticking (blue) times for $\mathrm{CH}_{4}(v=0) / \mathrm{Pt}(110)-(2 \times 1)$ at $T_{\mathrm{s}}=120 \mathrm{~K}$ and $E_{\mathrm{i}}=$ $0.2 \mathrm{eV}$ obtained in QCT calculations. (b) Same as (a) but for $\mathrm{CH}_{4}\left(\nu_{3}\right.$ $=1$ ).

we have restricted the $x$-axis to 100 ps, simply to help the visualization and comparison of the two time distributions. Whereas many molecules follow a slow and continuous trapping-desorption mechanism characterized by long interaction times, dissociation events take place only within the first $\sim 2$ ps. These results show that for long-time trapped molecules, the rate coefficient of desorption is much larger than that of dissociative chemisorption.

Interestingly, the absence of any dissociation event after 2 ps shows that over the full energy range considered in this work, long interaction times do not entail artificial reactive events due to leakage from vibrational motion to the reaction coordinate. This might be because molecules trapped for long times remain relatively far from the surface in the region of the physisorption well (see below) where coupling of vibrational modes with the reaction coordinate is weak. Inclusion of energy transfer to the surface due to electron-hole pair excitations in the theoretical model ${ }^{20,59}$ is not likely to entail reactive events detrimental to desorption. Finally, the monotonously (and sharply) increasing $E_{\mathrm{i}}$-dependence of the experimental $S_{0}$ data for $\mathrm{CH}_{4}(v=0)$ at $T_{\mathrm{s}}=120 \mathrm{~K}$ does not suggest a prominent role of surface defects in the energy range considered in this work. Thus, our analysis indicates that the comparison of experiments with the computed sticking probabilities for $T_{\mathrm{s}}=120 \mathrm{~K}$ could be well justified not only for $E_{\mathrm{i}} \gtrsim 0.5 \mathrm{eV}$ but in the full energy range considered in Figure 9b. Unfortunately, elucidating why the computed $S_{0}$ values for $\mathrm{CH}_{4}(\mathrm{v}=0)$ agree quantitatively with experiments only for $E_{\mathrm{i}} \leq 0.4 \mathrm{eV}$ and $T_{\mathrm{s}}=120 \mathrm{~K}$ is at present not clear and demands further investigation.

To understand why methane molecules trapped for long times on a defect-free $\mathrm{Pt}(110)-(2 \times 1)$ surface do not dissociate, it is interesting to characterize the state of such molecules. Figure 10 shows that after 100 ps the trapped molecules are almost all located over the valley sites where the physisorption well is the deepest $(\sim 0.1 \mathrm{eV}$ deeper than on the ridge, as shown in Figure 3). Methane dissociation on $\mathrm{Pt}(110)-(2 \times 1)$ is an activated process characterized by a TS whose energy is $\sim 0.6 \mathrm{eV}$ (with respect to the reference configuration of the molecule far from the surface). Therefore, (similar to many other highly activated systems) for physisorbed molecules, the lowest dissociation barrier is significantly larger than the barrier for desorption and then, the latter channel tends to prevail. However, in this case there is another, perhaps unusual geometric factor that also favors desorption over dissociation: physisorbed molecules remain most of the time over valley sites where not only the physisorption well is the deepest but also the energy barrier for dissociation is the highest (likely to be more than $1 \mathrm{eV}$ higher than on ridge $\mathrm{Pt}$ atoms ${ }^{23,24}$ ).

Finally, it is interesting to point out that the nonreactive character of trapped methane molecules on $\operatorname{Pt}(110)-(2 \times 1)$ in the energy range considered here (i.e., $\left.E_{\mathrm{i}} \gtrsim 0.2 \mathrm{eV}\right)$ provides an explanation of why $S_{0}$ can be computed using the RPH method of ref 23 despite assuming that molecules do not move laterally during the collision with the surface. Though many molecules are trapped and extensively explore the surface, they eventually desorb and do not contribute to $S_{0}$. All of the molecules that dissociate do it near the impact site through a rather direct mechanism.

\section{CONCLUSIONS}

We report initial dissociative sticking probabilities for $\mathrm{CH}_{4}$ and $\mathrm{CHD}_{3}$ on $\mathrm{Pt}(110)-(2 \times 1)$ obtained by QCT calculations and measured in supersonic molecular beam (SMB) experiments. QCT calculations make use of a reaction specific reactive force field (RFF) obtained by fitting of density functional theory (DFT) total energies, plus an empirical long-distance correction to account for van der Waals interactions. QCT and SMB data agree qualitatively over a wide range of translational energies, $0.2 \mathrm{eV} \leq E_{\mathrm{i}} \leq 1.3 \mathrm{eV}$, and surface temperatures: $T_{s}=650$ and $120 \mathrm{~K}$, for $\mathrm{CH}_{4}$ and $\mathrm{CHD}_{3}$, respectively, in the ground vibrational state $\mathrm{v}=0$, and for $\mathrm{CH}_{4}$ with one quantum of antisymmetric $\mathrm{C}-\mathrm{H}$ stretch excitation $\left(\nu_{3}=1\right)$. However, the calculated sticking probabilities are mostly lower than the experimental results by a factor of $\sim 2-$ 3. This underestimation is similar to the one obtained by Chadwick et al. in density functional molecular dynamics (DFMD) calculations for $\mathrm{CHD}_{3}$ for $E_{\mathrm{i}} \sim 1 \mathrm{eV}$ and $T_{\mathrm{s}}=650 \mathrm{~K}$ using the SRP approach. ${ }^{24}$ The fact that in the latter work the trajectories had been integrated only during $1 \mathrm{ps}$, and that after 1 ps a non-negligible fraction of molecules remain trapped near 

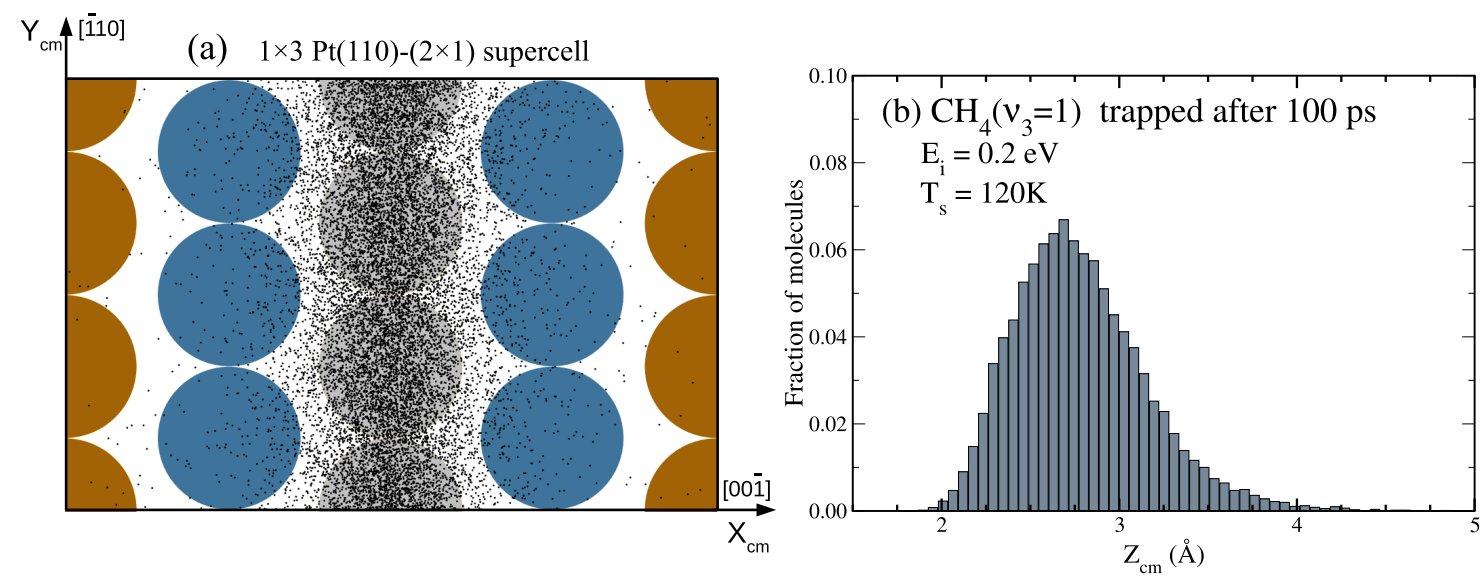

Figure 10. Position of the center-of-mass of trapped $\mathrm{CH}_{4}\left(\nu_{3}=1\right)$ molecules on $\mathrm{Pt}(110)-(2 \times 1)\left(E_{\mathrm{i}}=0.2 \mathrm{eV}, T_{\mathrm{s}}=120 \mathrm{~K}\right)$ at $t=100 \mathrm{ps}$. $(\mathrm{a})$ Black dots indicate the $X_{\mathrm{cm}}$ and $Y_{\mathrm{cm}}$ coordinates (parallel to the surface) within the $1 \times 3$ supercell. Yellow, blue, and gray circles represent the ridge, facet, and valley $\mathrm{Pt}$ atoms, respectively. (b) Distribution of the $Z$ coordinate of the molecular center-of-mass, $Z_{\mathrm{cm}}$, which reaches its maximum for $Z_{\mathrm{cm}} \sim$ $2.7 \AA$ where the physisorption well is the deepest (see Figure 3).

the surface, opened the question if the discrepancy with experiments might be due to molecules dissociating after more than 1 ps interaction times. In good agreement with the latter work, our QCT calculations predict significant trapping of methane on $\mathrm{Pt}(110)-(2 \times 1)$, with interaction times larger than 1 ps even for initial impact energies, $E_{\mathrm{i}}$, approaching $1 \mathrm{eV}$. Thanks to the possibility of following the time evolution of trapped molecules for much longer interaction times, we have shown that the fraction of trapped molecules and their typical interaction times increase dramatically when $E_{\mathrm{i}}$ and $T_{\mathrm{s}}$ decrease. However, in the whole range of impact energies considered for $T_{\mathrm{s}}=650 \mathrm{~K}$, and for $E_{\mathrm{i}} \gtrsim 0.5 \mathrm{eV}$ at $T_{\mathrm{s}}=120 \mathrm{~K}$, the contribution to the reactive sticking channel coming from long-interaction-time molecules is negligible. Such molecules eventually desorb intact due to an energy barrier to desorption much lower than to dissociation, and also because they tend to spend more time on top of the valley Pt atoms (where the physisorption well is the deepest), which offer the highest energy barrier toward dissociation. For $T_{\mathrm{s}}=120 \mathrm{~K}$, our analysis of the dynamics restricted to interaction times $\leq 1000$ ps suggests that the conclusions reached for $E_{\mathrm{i}} \gtrsim 0.5 \mathrm{eV}$ might also be valid for lower impact energies. However, this must be considered with caution because for $0.2 \mathrm{eV} \leq E_{\mathrm{i}} \lesssim 0.5 \mathrm{eV}$, the fraction of trapped molecules increases significantly and their lifetime reaches values beyond the scope of our standard molecular dynamics simulations. Unfortunately, the origin of the present underestimation of experiments (similar to the one obtained by Chadwick et al. in DFMD calculations for $\mathrm{CHD}_{3}$ and $T_{s}=650 \mathrm{~K}$ using the SRP approach ${ }^{24}$ ), is still unclear and requires further investigation.

\section{ASSOCIATED CONTENT}

\section{(s) Supporting Information}

The Supporting Information is available free of charge at https://pubs.acs.org/doi/10.1021/acs.jpcc.1c00807.

Reactive force field for methane interacting with $\operatorname{Pt}(110)-(2 \times 1)$ and RFF parameters for the $\mathrm{CH}_{4} /$ $\mathrm{Pt}(110)-(2 \times 1)$ system (PDF)

\section{AUTHOR INFORMATION}

\section{Corresponding Authors}

R. D. Beck - Laboratoire de Chimie Physique Moléculaire (LCPM), Ecole Polytechnique Fédérale de Lausanne (EPFL), 1015 Lausanne, Switzerland; $\odot$ orcid.org/0000-00028152-8290; Email: Rainer.Beck@epfl.ch

H. F. Busnengo - Grupo de Fisicoquímica en Interfases y Nanoestructuras, Instituto de Física Rosario (IFIR), CONICET-UNR, 2000 Rosario, Argentina; Facultad de Ciencias Exactas, Ingeniería y Agrimensura, Universidad Nacional de Rosario, 2000 Rosario, Argentina; $\odot$ orcid.org/ 0000-0002-7532-8495; Phone: +54 (0)341 - 4853200/22; Email: busnengo@ifir-conicet.gov.ar

\section{Authors}

I. F. Peludhero - Grupo de Fisicoquímica en Interfases y Nanoestructuras, Instituto de Física Rosario (IFIR), CONICET-UNR, 2000 Rosario, Argentina; Facultad de Ciencias Exactas, Ingeniería y Agrimensura, Universidad Nacional de Rosario, 2000 Rosario, Argentina

A. Gutiérrez-González - Laboratoire de Chimie Physique Moléculaire (LCPM), École Polytechnique Fédérale de Lausanne (EPFL), 1015 Lausanne, Switzerland

W. Dong - Université de Lyon, CNRS, École Normale Supérieure de Lyon, Université Lyon 1, Laboratoire de Chimie, UMR 5182, 69364 Lyon Cedex 07, France; College of Chemistry and Chemical Engineering, Hunan University, 410082 Changsha, China; $\odot$ orcid.org/0000-0003-37731029

Complete contact information is available at: https://pubs.acs.org/10.1021/acs.jpcc.1c00807

\section{Author Contributions}

I.F.P. performed the DFT calculations, parametrized the RFF, and made the QCT simulations using/modifying codes developed previously by W.D. and H.F.B. who have (both) supervised the calculations. A.G.-G. performed the experiments with and under the supervision of R.D.B. All of the authors participated in the discussion of the results and writing and/or revision of the manuscript.

\section{Notes}

The authors declare no competing financial interest. 


\section{ACKNOWLEDGMENTS}

This work has been supported by the Consejo Nacional de Investigationes Cientificas Técnicas (CONICET) and Ministerio de Educación, Cultura, Ciencia y Tecnología (ME) of Argentina and the Swiss National Science Foundation under the Argentinian-Swiss Joint Research Program (SNSF) Project No. IZSAZ2-173328 as well as the ANPCyT Project PICT No. 2750 (ME-Argentina), and the UNR PID projects ING534 and 80020180100121 UR. I.F.P. and H.F.B. acknowledge the computer time provided by CCT-Rosario Computational Center, and Centro de Simulación Computacional para Aplicaciones Tecnológicas (CSC), members of the HighPerformance Computing National System (SNCAD, MEArgentina).

\section{REFERENCES}

(1) Chorkendorff, I.; Niemantsverdriet, J. Concepts of Modern Catalysis and Kinetics, 2nd revised and enlarged edition; Wiley, 2007.

(2) Juurlink, L.; Killelea, D.; Utz, A. State-resolved probes of methane dissociation dynamics. Prog. Surf. Sci. 2009, 84, 69-134.

(3) Utz, A. L. Mode selective chemistry at surfaces. Curr. Opin. Solid State Mater. Sci. 2009, 13, 4-12.

(4) Chadwick, H.; Beck, R. D. Quantum state resolved gassurface reaction dynamics experiments: a tutorial review. Chem. Soc. Rev. 2016, 45, 3576-3594.

(5) Chadwick, H.; Beck, R. D. Quantum State-Resolved Studies of Chemisorption Reactions. Annu. Rev. Phys. Chem. 2017, 68, 39-61.

(6) Nave, S.; Tiwari, A. K.; Jackson, B. Dissociative Chemisorption of Methane on Ni and Pt Surfaces: Mode-Specific Chemistry and the Effects of Lattice Motion. J. Phys. Chem. A 2014, 118, 9615-9631.

(7) Jiang, B.; Yang, M.; Xie, D.; Guo, H. Quantum dynamics of polyatomic dissociative chemisorption on transition metal surfaces: mode specificity and bond selectivity. Chem. Soc. Rev. 2016, 45, $3621-3640$

(8) Bisson, R.; Sacchi, M.; Beck, R. D. State-resolved reactivity of $\mathrm{CH}_{4}$ on $\mathrm{Pt}(110)-(1 \times 2)$ : The role of surface orientation and impact site. J. Chem. Phys. 2010, 132, 094702.

(9) Bisson, R.; Sacchi, M.; Beck, R. D. Mode-specific reactivity of $\mathrm{CH}_{4}$ on $\mathrm{Pt}(110)-(1 \times 2)$ : The concerted role of stretch and bend excitation. Phys. Rev. B 2010, 82, No. 121404.

(10) Migliorini, D.; Chadwick, H.; Nattino, F.; Gutiérrez-González, A.; Dombrowski, E.; High, E. A.; Guo, H.; Utz, A. L.; Jackson, B.; Beck, R. D.; et al. Surface Reaction Barriometry: Methane Dissociation on Flat and Stepped Transition-Metal Surfaces. J. Phys. Chem. Lett. 2017, 8, 4177-4182.

(11) Cao, K.; Füchsel, G.; Kleyn, A. W.; Juurlink, L. B. F. Hydrogen adsorption and desorption from $\mathrm{Cu}(111)$ and $\mathrm{Cu}(211)$. Phys. Chem. Chem. Phys. 2018, 20, 22477-22488.

(12) Gutiérrez-González, A.; Crim, F. F.; Beck, R. D. Bond selective dissociation of methane $\left(\mathrm{CH}_{3} \mathrm{D}\right)$ on the steps and terraces of $\mathrm{Pt}(211)$. J. Chem. Phys. 2018, 149, 074701.

(13) Füchsel, G.; Cao, K.; Er, S.; Smeets, E. W. F.; Kleyn, A. W.; Juurlink, L. B. F.; Kroes, G.-J. Anomalous Dependence of the Reactivity on the Presence of Steps: Dissociation of D2 on $\mathrm{Cu}(211)$. J. Phys. Chem. Lett. 2018, 9, 170-175.

(14) Chadwick, H.; Gutirrez-Gonzlez, A.; Beck, R. D.; Kroes, G.-J. $\mathrm{CHD}_{3}$ Dissociation on the Kinked Pt(210) Surface: A Comparison of Experiment and Theory. J. Phys. Chem. C 2019, 123, 14530-14539.

(15) Smeets, E. W. F.; Füchsel, G.; Kroes, G.-J. Quantum Dynamics of Dissociative Chemisorption of $\mathrm{H} 2$ on the Stepped $\mathrm{Cu}(211)$ Surface. J. Phys. Chem. C 2019, 123, 23049-23063.

(16) Zhou, X.; Zhang, Y.; Guo, H.; Jiang, B. Towards bridging the structure gap in heterogeneous catalysis: the impact of defects in dissociative chemisorption of methane on Ir surfaces. Phys. Chem. Chem. Phys. 2021, 23, 4376-4385.

(17) Nave, S.; Jackson, B. Methane Dissociation on Ni(111): The Role of Lattice Reconstruction. Phys. Rev. Lett. 2007, 98, No. 173003.
(18) Nave, S.; Jackson, B. Methane dissociation on Ni(111): The effects of lattice motion and relaxation on reactivity. J. Chem. Phys. 2007, 127, No. 224702.

(19) Nattino, F.; Migliorini, D.; Kroes, G.-J.; Dombrowski, E.; High, E. A.; Killelea, D. R.; Utz, A. L. Chemically Accurate Simulation of a Polyatomic Molecule-Metal Surface Reaction. J. Phys. Chem. Lett. 2016, 7, 2402-2406.

(20) Luo, X.; Jiang, B.; Juaristi, J. I.; Alducin, M.; Guo, H. Electronhole pair effects in methane dissociative chemisorption on $\mathrm{Ni}(111)$. J. Chem. Phys. 2016, 145, No. 044704.

(21) Shen, X. J.; Lozano, A.; Dong, W.; Busnengo, H. F.; Yan, X. H. Towards Bond Selective Chemistry from First Principles: Methane on Metal Surfaces. Phys. Rev. Lett. 2014, 112, No. 046101.

(22) Lozano, A.; Shen, X. J.; Moiraghi, R.; Dong, W.; Busnengo, H. Cutting a chemical bond with demon's scissors: Mode- and bondselective reactivity of methane on metal surfaces. Surf. Sci. 2015, 640, 25-35. Reactivity Concepts at Surfaces: Coupling Theory with Experiment.

(23) Han, D.; Nave, S.; Jackson, B. Dissociative Chemisorption of Methane on Pt(110)-(12): Effects of Lattice Motion on Reactions at Step Edges. J. Phys. Chem. A 2013, 117, 8651-8659.

(24) Chadwick, H.; Gutiérrez-González, A.; Beck, R. D.; Kroes, G.-J. Transferability of the SRP32-vdW specific reaction parameter functional to $\mathrm{CHD}_{3}$ dissociation on $\mathrm{Pt}(110)-(2 \times 1)$. J. Chem. Phys. 2019, 150, No. 124702.

(25) Blöchl, P. E. Projector augmented-wave method. Phys. Rev. B 1994, 50, 17953-17979.

(26) Kresse, G.; Hafner, J. Ab initio Molecular Dynamics for Liquid Metals. Phys. Rev. B 1993, 47, 558-561.

(27) Kresse, G.; Hafner, J. Ab initio molecular-dynamics simulation of the liquid-metal-amorphous-semiconductor transition in germanium. Phys. Rev. B 1994, 49, 14251-14269.

(28) Kresse, G.; Furthmüller, J. Efficiency of Ab-Initio Total Energy Calculations for Metals and Semiconductors Using a Plane-Wave Basis Set. Comput. Mater. Sci. 1996, 6, 15-50.

(29) Kresse, G.; Furthmüller, J. Efficient Iterative Schemes for Ab Initio Total-Energy Calculations Using a Plane-Wave Basis Set. Phys. Rev. B 1996, 54, 11169-11186.

(30) Kresse, G.; Joubert, D. From ultrasoft pseudopotentials to the projector augmented-wave method. Phys. Rev. B 1999, 59, 17581775.

(31) Perdew, J. P.; Burke, K.; Ernzerhof, M. Generalized Gradient Approximation Made Simple. Phys. Rev. Lett. 1996, 77, 3865-3868.

(32) Arblaster, J. W. Crystallographic Properties of Platinum. Platinum Met. Rev. 2006, 50, 118-119.

(33) Nave, S.; Tiwari, A. K.; Jackson, B. Methane dissociation and adsorption on $\mathrm{Ni}(111), \operatorname{Pt}(111), \mathrm{Ni}(100), \mathrm{Pt}(100)$, and $\mathrm{Pt}(110)$ (1×2): Energetic study. J. Chem. Phys. 2010, 132, No. 054705.

(34) Wong, S. S. M. Computational Methods in Physics and Engineering, 2nd ed.; World Scientific, Singapore, 1997.

(35) Lozano, A.; Shen, X.; Moiraghi, R.; Peludhero, I.; Ramos, M.; Dong, W.; Busnengo, $\mathrm{H}$. Reaction specific force fields for methane dissociation on metal surfaces. in preparation.

(36) Verlet, L. Computer "Experiments" on Classical Fluids. I. Thermodynamical Properties of Lennard-Jones Molecules. Phys. Rev. 1967, 159, 98-103.

(37) Verlet, L. Computer "Experiments" on Classical Fluids. II. Equilibrium Correlation Functions. Phys. Rev. 1968, 165, 201-214.

(38) Sewell, T. D.; Thompson, D. L. Classical Trajectory Methods for Polyatomic Molecules. Int. J. Mod. Phys. B 1997, 11, 1067-1112.

(39) Walker, A. V.; King, D. A. Dynamics of the Dissociative Adsorption of Methane on Pt(110)-(1×2). Phys. Rev. Lett. 1999, 82, 5156-5159.

(40) Jackson, B. Direct and trapping-mediated pathways to dissociative chemisorption: $\mathrm{CH}_{4}$ dissociation on $\operatorname{Ir}(111)$ with step defects. J. Chem. Phys. 2020, 153, No. 034704.

(41) Perez-Sala, X.; Igual, L.; Escalera, S.; Angulo, C. Robotic Vision: Technologies for Machine Learning and Vision Applications; García- 
Rodriguez, J.; García-Rodriguez, J.; Cazorla-Quevedo, M. A., Eds.; IGI Global, 2012; pp 23-42.

(42) Chen, L.; Ueta, H.; Bisson, R.; Beck, R. D. Quantum stateresolved gas/surface reaction dynamics probed by reflection absorption infrared spectroscopy. Rev. Sci. Instrum. 2013, 84, No. 053902.

(43) Scoles, G. Atomic and Molecular Beam Methods, v. 1; Oxford University Press, 1988.

(44) Chadwick, H.; Guo, H.; Gutirrez-Gonzlez, A.; Menzel, J. P.; Jackson, B.; Beck, R. D. Methane dissociation on the steps and terraces of $\mathrm{Pt}(211)$ resolved by quantum state and impact site. J. Chem. Phys. 2018, 148, No. 014701.

(45) Gutiérrez-González, A.; Torio, M.; Busnengo, H.; Beck, R. Site Selective Detection of Methane Dissociation on Stepped Pt Surfaces. Top. Catal. 2019, 62, 859-873.

(46) Gutiérrez-González, A.; Beck, R. D. Quantum state and surfacesite-resolved studies of methane chemisorption by vibrational spectroscopies. Phys. Chem. Chem. Phys. 2020, 22, 17448-17459.

(47) King, D. A.; Wells, M. G. Molecular beam investigation of adsorption kinetics on bulk metal targets: Nitrogen on tungsten. Surf. Sci. 1972, 29, 454-482.

(48) Fery, P.; Moritz, W.; Wolf, D. Structure determination of the $(1 \times 2)$ and $(1 \times 3)$ reconstructions of $\mathrm{Pt}(110)$ by low-energy electron diffraction. Phys. Rev. B 1988, 38, 7275-7286.

(49) Sowa, E. C.; van Hove, M. A.; Adams, D. L. The missing-row model for the reconstructed $\mathrm{Pt}(110)(1 \times 2)$ surface: A leed intensity analysis showing multilayer distortions. Surf. Sci. 1988, 199, 174-182.

(50) Fenter, P.; Gustafsson, T. Structural analysis of the Pt(110)$(1 \times 2)$ surface using medium-energy ion scattering. Phys. Rev. B 1988, 38, 10197-10204.

(51) Vlieg, E.; Robinson, I.; Kern, K. Relaxations in the missing-row structure of the $(1 \times 2)$ reconstructed surfaces of $\mathrm{Au}(110)$ and Pt(110). Surf. Sci. 1990, 233, 248-254.

(52) Venuti, E.; Halonen, L.; della Valle, R. G. High dimensional anharmonic potential energy surfaces: The case of methane. J. Chem. Phys. 1999, 110, 7339-7347.

(53) Lozano, A.; Groß, A.; Busnengo, H. F. Phys. Chem. Chem. Phys. 2009, 11, 5814.

(54) Mastromatteo, M.; Jackson, B. The dissociative chemisorption of methane on $\mathrm{Ni}(100)$ and $\mathrm{Ni}(111)$ : Classical and quantum studies based on the reaction path Hamiltonian. J. Chem. Phys. 2013, 139, No. 194701.

(55) Guo, H.; Jackson, B. Methane dissociation on stepped Ni surfaces resolved by impact site, collision energy, vibrational state, and lattice distortion. J. Chem. Phys. 2019, 150, No. 204703.

(56) Moiraghi, R.; Lozano, A.; Peterson, E.; Utz, A.; Dong, W.; Busnengo, H. F. Nonthermalized Precursor-Mediated Dissociative Chemisorption at High Catalysis Temperatures. J. Phys. Chem. Lett. 2020, 11, 2211-2218.

(57) Gutiérrez-González, A. Bond-selective and surface-site-specific dissociation of methane on platinum. Ph. D. Thesis, Cole Polytechnique Fdrale de Lausanne, 2019.

(58) Busnengo, H. F.; Crespos, C.; Dong, W.; Rayez, J. C.; Salin, A. Classical dynamics of dissociative adsorption for a nonactivated system: The role of zero point energy. J. Chem. Phys. 2002, 116, 9005-9013.

(59) Alducin, M.; Díez Muiño, R.; Juaristi, J. Non-adiabatic effects in elementary reaction processes at metal surfaces. Prog. Surf. Sci. 2017, 92, 317-340. 